\title{
Beyond injecting drug use: investigation of a Victorian cluster of hepatitis $C$ among HIV-infected men who have sex with men
}

\section{Andrew A Mahony MB BS, FRACP Infectious Diseases Physician,' and Public Health Registrar ${ }^{2}$ \\ Ellen J Donnan MAppEpid, MPH, BNurs, Epidemiologist Communicable Diseas Prevention and Control Unit ${ }^{2}$ \\ Rosemary A Lester MB BS, MPH, FAFPHM Chief Health Officer \\ Joseph S Doyle MB BS, MSC, FRACP Infectious Diseases Physician $^{3,4}$ \\ James Knox MB BS, BSc, DTMH Infectious Diseases Registrar \\ Samantha L Tracy GradDipIntern\&WebComp Hospital Scientist, Grade2 \\ Scott Bowden \\ $\mathrm{PhD}$, Senior Scientist Molecular Microbiology \\ Joseph J Sasadeusz MB BS, FRACP, PhD Infectious Disease Physician ${ }^{5,6}$ \\ 1 Austin Health, Melbourne, VIC. \\ 2 Department of Health Melbourne, VIC \\ 3 Victorian Infectious Diseases Reference Laboratory, Melbourne, VIC \\ 4 Burnet Institute Melbourne, VIC \\ 5 Victorian Infectious Diseases Service Melbourne Health, Melbourne, VIC. 6 Alfred Health Melbourne, VIC. \\ andrew.mahony@ austin.org.au}

MJA 2013; 198: 210-214 doi: 10.5694/mjal2.10556 epatitis C virus (HCV) has emerged worldwide as a major infectious disease. An estimated 221000 Australians (about 1\% of the population) were living with chronic hepatitis C by the end of 2010, and HCV infection is now the most common indication for liver transplantation. ${ }^{1,2}$ While HCV diagnosis rates have declined nationally over the past 10 years, the persistence of risk behaviours, and the relatively limited uptake of $\mathrm{HCV}$ treatment (only 3760 Australians were treated for HCV infection in 2010) suggest that the epidemic is ongoing. ${ }^{1}$

Public health measures to curb $\mathrm{HCV}$ infection in Australia have focused on harm reduction. Injecting drug use (IDU) remains the predominant risk factor, ${ }^{3}$ but there has been accumulating evidence of sexual (permucosal) transmission over the past decade. Two per cent of the 899 newly acquired Australian cases from 1997 to 2000 were considered related to sexual contact, ${ }^{4}$ and more recent recognition of HCV-HIV co-infection in men who have sex with men (MSM) has prompted reconsideration of what constitutes blood-to-blood contact. ${ }^{5-7}$ Several studies of HIV-infected MSM have identified specific risk factors for HCV transmission: group sex, unprotected intercourse, fisting (inserting a hand into the rectum), non-injecting (ie, nasal or rectal) drug use, use of sex toys, and concurrent genital ulceration. ${ }^{6,8-10}$ Notably, reports of sexually transmitted $\mathrm{HCV}$ in HIV-negative MSM remain rare. Nonetheless, with the accelerated progression of liver fibrosis in patients co-infected with HCV-HIV now well established, coinfection has become a key issue, with liver disease-related mortality ranking as one of the leading causes of death among HIV-infected individuals in the developed world. ${ }^{11,12}$

The prevalence of $\mathrm{HCV}$ co-infection in Australians living with HIV was estimated at $13.1 \%$ in 2002 and $9.9 \%$ in 2010, with most co-infected individuals reporting IDU as a risk fac-

Objectives: To examine increased notifications of hepatitis $\mathrm{C}$ virus (HCV) in men who have sex with men (MSM) infected with HIV in Victoria, and evaluate HCV transmission risk factors other than injecting drug use.

Design, setting and participants: Case series through retrospective review of all HCV cases in Victoria from 1 April 2010 to 30 June 2011, with clinical and laboratory data examined in likely MSM to identify a co-infected cohort. Patients with newly acquired HCV with HIV co-infection were invited to complete a questionnaire exploring novel risk factors for $\mathrm{HCV}$ transmission (non-injecting drug use, sexual practices with increased likelihood of trauma, and presence of genital ulcers). Sequencing was performed to determine the local molecular epidemiology of HCV co-infection.

Main outcome measures: Demographics of newly co-infected MSM, traditional versus novel risk factors for HCV acquisition, prior knowledge of potential for sexual transmission of HCV, and association between viral sequences.

Results: Thirty-one patients with HIV were identified from 3365 notifications of hepatitis C. The median age was 42 years, and median time from HIV to HCV diagnosis was 22 months. Most patients were asymptomatic, with abnormal liver function tests prompting HCV testing. Interviews with 14 patients identified a high prevalence of novel risk factors and limited knowledge of HCV risk. Two clusters of matching viral sequences were identified.

Conclusions: Novel HCV transmission routes have emerged in Victoria. These data reinforce the need for targeted testing and prevention strategies among HIV-infected MSM.

tor. ${ }^{1,13}$ One Melbourne sexual health clinic, however, identified 24 patients with newly acquired $\mathrm{HCV}$ without a history of IDU among 620 HIVinfected MSM over an 8-year period up to March 2010. ${ }^{14}$ We aimed to examine HCV acquisition among HIV-infected MSM in Victoria and to determine the relevance of novel risk factors within this group.

\section{Methods}

We undertook a retrospective review of all cases of HCV notified to the Victorian Department of Health from 1 April 2010 to 30 June 2011. The investigation began in April 2011, with subsequent cases examined prospectively. Hepatitis $C$ is a nationally notifiable disease; in Victoria, medical practitioners and laboratories are legally required to notify the Department of Health. From these notifications we delineated a case series of newly acquired HCV infection in MSM infected with HIV (Box 1).

High-caseload centres were the focus of our investigation, including general practitioner clinics specialising in HIV medicine and infectious diseases services. All notifications from these centres were crosschecked with the Victorian HIV Registry (Centre for Population Health, Burnet Institute, Melbourne) to identify patients with co-infection. We undertook the same process for any doctors' notifications where MSM behaviour was indicated. A standard definition of newly acquired HCV was used, based on any one of: antibody seroconversion in the previous 24 months, new viraemia with a negative antibody test in the previous 24 months, or unexplained alanine aminotransaminase (ALT) elevation (at least $350 \mathrm{U} / \mathrm{L}$; reference interval, 5-50 U/L) at first positive antibody or HCV RNA detection. ${ }^{15}$

For these patients, we gathered epidemiological data from the following sources:

- Enhanced surveillance data from notifications of hepatitis $\mathrm{C}$ by clinicians; ${ }^{16}$

- The Victorian Infectious Diseases Reference Laboratory (VIDRL) for $\mathrm{HCV}$ genotyping, HIV viral loads, CD4+ lymphocyte counts and results 
1 Process of identifying cases of newly acquired hepatitis C virus (HCV) in HIV-infected men who have sex with men (MSM) in Victoria from 1 April 2010 to 30 June 2011

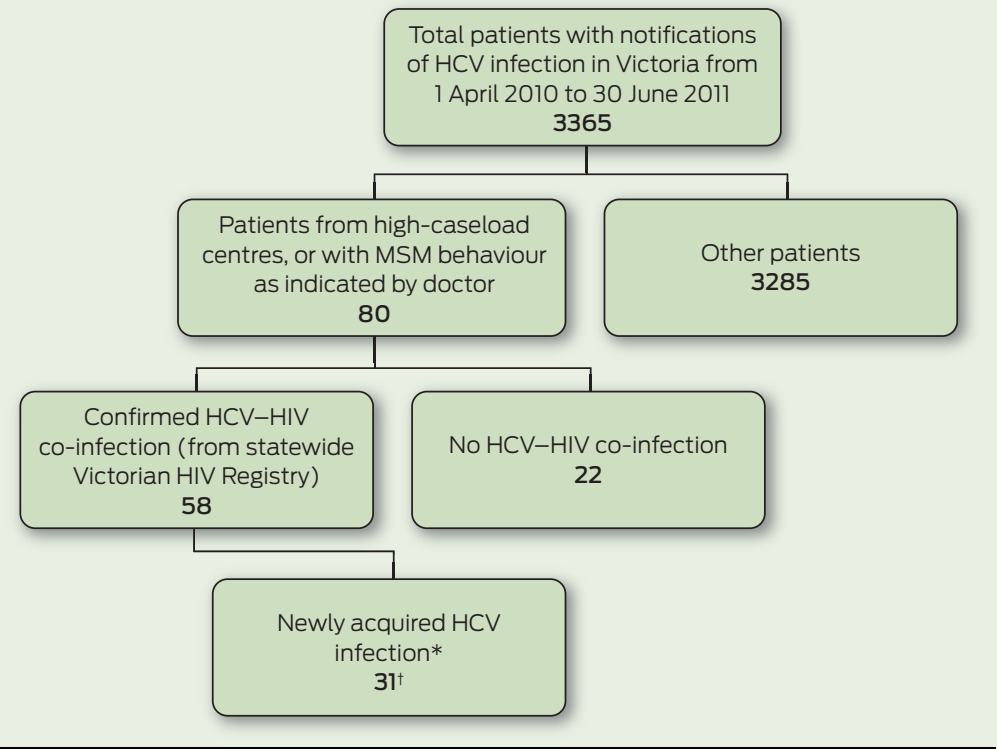

* We searched for previous HCV antibody results at four Victorian pathology services and the Victorian Infectious Diseases Reference Laboratory † Two patients had false-positive results of polymerase chain reaction (PCR) tests with normal liver function tests; the remaining 25 patients did not meet the seroconversion or alanine aminotransaminase (ALT) criteria.

of syphilis serology. We defined recent HIV monitoring as any results within 3 months of HCV diagnosis. We looked for syphilis serology results indicating infectiousness (a fourfold or greater rise in rapid plasma reagin titre plus a reactive specific treponemal test);

- The Department of Health Notifiable Infectious Diseases Surveillance database, to identify sexually trans- mitted infection (STI) notifications in the 12 months before hepatitis $\mathrm{C}$ notification;

- A cluster-specific questionnaire examining risk factors for $\mathrm{HCV}$ acquisition, which was designed by the authors, the Sexual Health and Viral Hepatitis team at the Department of Health, and the Partner Notification Officers (PNOs). Patients were first contacted by their treating doctor to seek informed consent. The PNOs then conducted the questionnaire by telephone with consenting patients who had been de-identified.

To examine whether specific strains of $\mathrm{HCV}$ were being transmitted, VIDRL staff performed sequence analysis of HCV isolates from a random selection of patients. The HCV genotype and subtype of samples were determined using a commercially available assay, VERSANT Hepatitis C Virus Genotype Assay (LiPA) version 2.0 (Siemens Healthcare Diagnostics). Further discrimination was carried out by sequence analysis of the HCV core region. ${ }^{17}$ Sequences were aligned with references using ClustalW and BioEdit (http:// www.mbio. ncsu.edu/BioEdit/ bioedit.html).

This study was approved as an investigation into an emerging outbreak of HCV in Victoria under the direction of the then Deputy Chief Health Officer (RL) in accordance with the Public Health and Wellbeing Act 2008 (Vic).

\section{Results}

Newly acquired HCV co-infection in MSM was identified in 31 patients (Box 1). Demographics of this group are shown in Box 2. The median ALT level at diagnosis was $580 \mathrm{U} / \mathrm{L}$ (range, $52-1715 \mathrm{U} / \mathrm{L})$. Nineteen patients (61\%) had HCV antibody seroconver-

2 Patient characteristics and results of investigations in 31 HIV-infected men who have sex with men who had newly acquired hepatitis C virus (HCV) infection in Victoria, 1 April 2010 to 30 June 2011

Patient characteristics and investigations

Median age of patients at time of diagnosis with HCV (range)

Region of residence

Patients residing in metropolitan areas (\%)

Patients residing in rural areas (\%)

Country of birth

Patients born in Australia (\%)

Patients born overseas (\%)

Reason for HCV testing

Patients with symptoms of acute hepatitis (\%)

Patients who were asymptomatic with abnormal results of liver function tests (\%) Median time between HIV and HCV diagnoses (range)

Median CD4+ lymphocyte cell count (range); and CD4 percentage of total lymphocytes around time of HCV diagnosis (range)

Median HIV viral load around time of HCV diagnosis (range)

Patients who reported injecting drugs ever (\%)

Patients who reported having sexual contact with a known HCV-positive partner (\%)
Results

42 years (26-57 years)

$27(87 \%)$

$4(13 \%)$

$27(87 \%)$

$4(13 \%)$

$3(10 \%)$

$28(90 \%)$

22 months ( 2 months - 17 years)*

$535 / \mu \mathrm{L}(198-822 / \mu \mathrm{L})$;

$25 \%{ }^{\dagger}(16 \%-42 \%)$

$<50$ copies/mL

$(<50 \text { to }>100000 \text { copies } / \mathrm{mL})^{\dagger}$

$12(39 \%)$

$10(32 \%)$

* Three patients were outside Victoria at the time of their HIV diagnosis, so the time between HIV and HCV diagnoses could not be determined.

† Two patients had not had recent HIV monitoring in Victoria at the time of their HCV diagnosis. 
3 Sexually transmitted infections (STIs) acquired between HIV and hepatitis C virus (HCV) diagnoses by 31 HIVinfected men who have sex with men who had newly acquired HCV infection in Victoria

\begin{tabular}{lcc} 
STI* & $\begin{array}{c}\text { Total patients with } \\
\text { STIs diagnosed } \\
\text { after HIV diagnosis }\end{array}$ & $\begin{array}{c}\text { Patients with STIs } \\
\text { diagnosed in the 12 } \\
\text { months before HCV } \\
\text { diagnosis }\end{array}$ \\
\hline $\begin{array}{l}\text { Any notifiable sexually } \\
\text { transmitted infection }\end{array}$ & 19 & 15 \\
Chlamydia trachomatis & 14 & 7 \\
Neisseria gonorrhoeae & 10 & 7 \\
Syphilis, infectious & 8 & $4^{\dagger}$ \\
\hline
\end{tabular}

* None of the patients had been diagnosed with chancroid, donovanosis or hepatitis $A$, and one patient had been diagnosed with hepatitis $B$. $†$ Syphilis serology tests were done for 26 patients in the 12 months before HCV

diagnosis. ticipated in our questionnaire and had not injected drugs in the 12 months before diagnosis with $\mathrm{HCV}$.

\section{Discussion}

This study described the epidemiology of acute HCV in HIV-infected MSM in Victoria and confirmed the importance of transmission risk factors other than IDU. This has direct relevance to testing strategies and prevention messages for HIV-infected MSM.

The 31 cases of newly acquired $\mathrm{HCV}$ co-infection represent a relatively small proportion $(0.9 \%)$ of the total HCV cases statewide. However, among the estimated 5722 Victorians living with HIV at the end of 2010 (about $0.1 \%$ of the population), 31 cases of $\mathrm{HCV}$ is disproportionately high. ${ }^{18,19}$ While other Australian studies have reported instances of HIV$\mathrm{HCV}$ co-infection - particularly the Australian Trial in Acute Hepatitis C (ATAHC), with 50 cases nationwide, 23 in MSM with sexual exposure ${ }^{20}$ the density of cases in our study's single jurisdiction is concerning. The sequencing results support the hypothesis that overlapping risk factors contributed to HCV transmission, and related sequences suggest that at least two common lineages of $\mathrm{HCV}$ are circulating in this group.

Within this case series, IDU was a relatively uncommon risk factor for HCV acquisition, with $39 \%$ of the 31 men reporting any previous history of IDU, and five of 14 patients interviewed confirming IDU in the 12 months before HCV diagnosis. Con- versely, sexual risk factors were highly prevalent. Inconsistent condom use with multiple sexual partners was universal among the men who completed the questionnaire, and correlates with the high proportion of STIs in the cluster (15/31 patients). Unprotected sex has become the paradoxical reaction to the success story of anti-retroviral therapy (ART). ${ }^{21}$ Potentially traumatic sexual practices combined with noninjecting drug use were frequently reported and were probably synergistic in HCV transmission. ${ }^{6}$ While it is likely that IDU introduces HCV into a sexual network, there is now evidence that sexual transmission in HIV-infected MSM does occur, irrespective of CD4+ lymphocyte counts. ${ }^{9,10,20,22}$ Serosorting, or selection of sexual partners on the basis of their HIV status, then concentrates HCV transmission within this population.

This study reaffirms the need for periodic HCV testing in HIV-infected MSM, particularly where history-taking reveals high-risk sexual behaviours. Based on our study, we would also argue that any diagnosis of STI in this group should prompt discussion around $\mathrm{HCV}$ transmission. Most cases were identified because abnormal LFTs were detected during routine management of ART. Given that $\mathrm{HCV}$ infection is commonly asymptomatic, there may be more MSM in the community with undiagnosed $\mathrm{HCV}$ who are not aware of the risk to sexual partners. Further, there is a risk of HCV re-infection after treatment or spontaneous clearance, necessitating continued HCV RNA least 10 patients). Only five patients had been aware of the potential for $\mathrm{HCV}$ to be sexually transmitted before their diagnosis with HCV. Twelve patients reported changing their sexual practices since being diagnosed.

HCV genotyping results were available for 29 patients. Genotype 1a was identified in 21 patients, genotype $3 \mathrm{a}$ in seven, and one patient had spontaneous clearance of virus. Nine test samples (six HCV genotype $1 \mathrm{a}$ and three genotype $3 \mathrm{a}$ ) were further characterised by sequencing of the HCV core region. Nucleotide sequence alignments showed that two of the HCV 1a samples shared $100 \%$ identity with each other, as did the three HCV $3 a$ isolates. At least one patient within each cluster par-

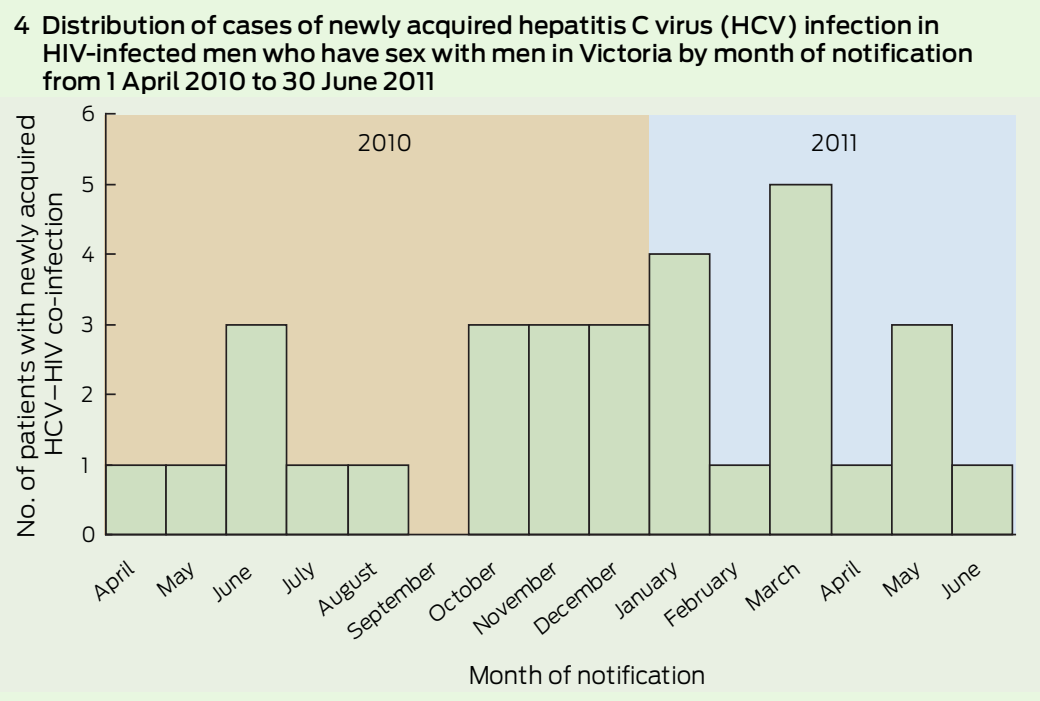


5 Risk factors for hepatitis C virus (HCV) acquisition in the 12 months before diagnosis in 14 HIV-infected men who have sex with men

Risk facto

\begin{tabular}{|c|c|}
\hline Injecting drug use & $5(36 \%)$ \\
\hline $\begin{array}{l}\text { Sharing of injecting paraphernalia (eg, syringe, tourniquet, spoon) (proportion } \\
\text { of injecting drug users) }\end{array}$ & $3(60 \%)$ \\
\hline \multicolumn{2}{|l|}{ Tattoo or piercing } \\
\hline Any & $6(43 \%)$ \\
\hline Performed overseas (proportion of those with tattoos or piercings) & $2(33 \%)$ \\
\hline Sexual contact with known HCV-positive partner & $4(29 \%)$ \\
\hline Sexual activity where blood visibly present & $3(21 \%)$ \\
\hline Serosorting (selection of sexual partner by HIV status) & $12(86 \%)$ \\
\hline \multicolumn{2}{|l|}{ Non-injecting drug use } \\
\hline Any & $11(79 \%)$ \\
\hline Ice & $4(29 \%)$ \\
\hline$\gamma$-hydroxybutyrate (GHB) & $4(29 \%)$ \\
\hline Cannabis & $4(29 \%)$ \\
\hline Non-injecting drug use before or during sexual activity & $11(79 \%)$ \\
\hline \multicolumn{2}{|l|}{ Number of sexual partners } \\
\hline 0 & 0 \\
\hline 1 & 0 \\
\hline $2-9$ & $4(29 \%)$ \\
\hline $10-29$ & $5(36 \%)$ \\
\hline$\geqslant 30$ & $5(36 \%)$ \\
\hline \multicolumn{2}{|l|}{ Condom use } \\
\hline Always & 0 \\
\hline Usually & $5(36 \%)$ \\
\hline Sometimes & $3(21 \%)$ \\
\hline Occasionally & $3(21 \%)$ \\
\hline Never & $3(21 \%)$ \\
\hline Group sex participant & $12(86 \%)$ \\
\hline \multicolumn{2}{|l|}{ Frequency of episodes of group sex in the 12 months before diagnosis } \\
\hline 0 & $2(14 \%)$ \\
\hline $1-2$ & $3(21 \%)$ \\
\hline $3-5$ & $3(21 \%)$ \\
\hline $6-10$ & $2(14 \%)$ \\
\hline$>10$ & $5(36 \%)$ \\
\hline Use of insertive sex toys & $10(71 \%)$ \\
\hline Sharing of toys between partners (proportion of those using insertive sex toys) & $7(70 \%)$ \\
\hline Use of condoms over sex toys (proportion of those using insertive sex toys) & $2(20 \%)$ \\
\hline Washing of toys between partners (proportion of those using insertive sex toys) & $2(20 \%)$ \\
\hline Fisting & 10 (71\%) \\
\hline
\end{tabular}

testing to detect possible re-infection. ${ }^{23}$ This highlights the need for effective education campaigns to circumvent repeated courses of therapy that are both toxic and expensive. There is a much higher response to early treatment with current $\mathrm{HCV}$ therapy even in HIV co-infected patients, which again emphasises the need for frequent testing and timely identification and treatment. ${ }^{24}$

This study has a number of limitations. It is possible that the casefinding strategy may have missed potential patients who underwent their HCV testing elsewhere. The increase in notifications of cases may have been the result of increased testing. An alert was issued by the Chief Health Officer advising of an increase in hepatitis C in HIV-positive men in May 2011, which may have contributed to this. ${ }^{25}$ The retrospective 12-month period of interest used for the questionnaire may have introduced error resulting from inaccuracy in patients' recollections of activities undertaken. Finally, only 14 patients participated in the questionnaire. While this group was representative of the cluster on demographic variables, we can only assume that this also applied to sexual risk factors.

There is a pressing need for the development and rollout of health promotion strategies to increase awareness of HCV sexual transmission and risk mitigation in Australia, in conjunction with the affected community. This is particularly important given that we as practitioners have previously generally delivered a message that $\mathrm{HCV}$ was not effectively sexually transmitted. We have continued to monitor the incidence of HCVHIV co-infection in Victoria, and are aware of at least 13 further cases in the second half of 2011 (data not shown). Prospective data collection should measure changes in risk behaviours in response to prevention campaigns. Without intervention, however, the tide of HCV-HIV coinfection has the potential to erode the revolutionary gains made in the health of HIV-infected men.

Acknowledgements: We thank the Partner Notification Officers at the Department of Health for their assistance in questionnaire design and delivery, and the highcaseload general practitioners who facilitated contact with patients.

Competing interests: No relevant disclosures.

Received 28 Mar 2012, accepted 11 Oct 2012.

1 The Kirby Institute. HIV, viral hepatitis and sexually transmissible infections in Australia. Annual surveillance report 2011. Sydney: The Kirby Institute, University of New South Wales, 2011.

2 Gidding HF, Topp L, Middleton M, et al. The epidemiology of hepatitis C in Australia: notifications, treatment uptake and liver transplantations, 1997-2006. J Gastroenterol Hepatol 2009: 24:1648-1654.

3 Guy R, Devadason D, Lim M, et al. Enhanced case detection for newly acquired hepatitis C infection: epidemiological findings and health service implications. Commun Dis Intell 2008; 32: $250-256$.

4 Robotin MC, Copland J, Tallis G, et al. Surveillance for newly acquired hepatitis C in Australia. J Gastroenterol Hepatol 2004; 19: 283-288.

5 Rauch A, Rickenbach M, Weber R, et al. Unsafe sex and increased incidence of hepatitis C virus infection among HIV-infected men who have sex with men: the Swiss HIV cohort study. Clin Infect Dis 2005; 41: 395-402.

6 Danta M, Brown D, Bhagani S, et al. Recent epidemic of acute hepatitis C virus in HIV-positive men who have sex with men linked to high-risk sexual behaviours. AIDS 2007; 21: 983-991.

7 Matthews GV, Hellard M, Kaldor J, et al. Further evidence of HCV sexual transmission among HIVpositive men who have sex with men: response to Danta et al. AIDS 2007; 21: 2112-2113.

8 van de $L$ aar TJ, van der Bij AK, Prins $M$, et al. Increase in HCV incidence among men who have sex with men in Amsterdam most likely caused by sexual transmission. J Infect Dis 2007; 196: 230-238.

9 Schmidt AJ, Rockstroh JK, Vogel M, et al. Trouble with bleeding: risk factors for acute hepatitis $C$ among HIV-positive gay men from Germany a case-control study. PLoS One 2011; 6: el7781.

10 Centers for Disease Control and Prevention (CDC). Sexual transmission of hepatitis C virus 
among HIV-infected men who have sex with men - New York City, 2005-2010. MMWR Morb Mortal Wkly Rep 2011; 60: 945-950.

11 Thein HH, Yi Q, Dore GJ, Krahn MD. Natural history of hepatitis C virus infection in HIV-infected individuals and the impact of HIV in the era of highly active antiretroviral therapy: a metaanalysis. AIDS 2008; 22: 1979-1991.

12 Weber R, Sabin CA, Friis-Møller N, et al. Liver-related deaths in persons infected with the human immunodeficiency virus: the D:A:D study. Arch Intern Med 2006; 166 : 1632-1641.

13 Lincoln D, Petoumenos K, Dore GJ; Australian HIV Observational Database. HIV/HBV and HIV/HCV coinfection, and outcomes following highly active antiretroviral therapy. HIV Med 2003; 4 : 241-249.

14 Gamage DG, Read TR, Bradshaw CS, et al. Incidence of hepatitis-C among HIV infected men who have sex with men (MSM) attending a sexual health service: a cohort study. BMC Infect Dis 2011; 11: 39.

15 Department of Health and Ageing Communicable Diseases Network Australia. Australian national notifiable diseases case definitions. Hepatitis C (newly acquired) case definition. http://www.health.gov.au/internet/ main/publishing.nsf/Content/cda-surveil-nndsscasedefs-cd_hepcnew.htm (accessed Apr 2011).

16 Victorian Government Department of Health, Communicable Disease Prevention and Control Unit. Notification of hepatitis C. Enhanced surveillance form. http://docs.health.vic.gov.au/ docs/doc/Notification-of-Hepatitis-C (accessed Jun 2011).

17 Dev AT, McCaw R, Sundararajan V, et al. Southeast Asian patients with chronic hepatitis C: the impact of novel genotypes and race on treatment outcome. Hepatology 2002; 36: 1259-1265.

18 Communicable Disease Prevention and Control Unit. Surveillance of notifiable infectious diseases in Victoria, 2010. Melbourne: Victorian Government Department of Health, in press.

19 Spatial Analysis and Research, Department of Planning and Community Development, Victorian Government. Victorian population bulletin 2011. Melbourne: Stream Solutions, 2011.

20 Matthews GV, Pham ST, Hellard M, et al; ATAHC Study Group. Patterns and characteristics of hepatitis C transmission clusters among HIVpositive and HIV-negative individuals in the
Australian Trial in Acute Hepatitis C. Clin Infect Dis 2011; 52: 803-811.

21 Guy RJ, Spelman T, Stoove M, et al. Risk factors for HIV seroconversion in men who have sex with men in Victoria, Australia: results from a sentinel surveillance system. Sex Health 2011; 8 : 319-329.

22 Taylor LE, Holubar M, Wu K, et al. Incident hepatitis C virus infection among US HIV-infected men enrolled in clinical trials. Clin Infect Dis 2011; 52: 812-818.

23 Lambers FA, Prins M, Thomas X, et al. Alarming incidence of hepatitis $C$ virus re-infection after treatment of sexually acquired acute hepatitis C virus infection in HIV-infected MSM. AIDS 2011; 25: F2l-F27.

24 Matthews GV, Hellard M, Haber P, et al; ATAHC Study Group. Characteristics and treatment outcomes among HIV-infected individuals in the Australian Trial in Acute Hepatitis C. Clin Infect Dis 2009; 48: 650-658.

25 Victorian Government Department of Health, Office of the Chief Health Officer. Hepatitis C increase in HIV positive men - May 2011. http:// www.health.vic.gov.au/chiefhealthofficer/alerts/ alert-2011-05-hepatitisc.htm (accessed Feb 2012). 